\title{
Synthesis and Imaging Performances of A New Type PAC
}

\author{
Wang Xuefei, Liu Zhengping, Gu Jiangnan and Yu Shangxian \\ Department of Chemistry, Beijing Normal University, Beijing, 100875, China
}

\begin{abstract}
In this paper, a new type photoactive compounds (PAC) of diazidonaphthoquinone(DNQ) have been synthesized based on the ballast of 2,2-diphenyl-2-phenols group acetic lectone compound which is synthesized from benzilic acid
\end{abstract}

The new type PACs have good miscibility with common novolacs and dissolubility in the solvents used in positive photoresists and photosensitive compositions for PS plates. The photosensitive compositions composed of each new PAC and the novolac have well film-forming performance. Before exposure, the formed photosensitive film behaves strong dissolution inhibiting effect in alkaline developer. After exposure it becomes very easy to dissolve in developer. In other words, it obtains good dissolution accelerating performance in exposed areas. Because the change of the effect of dissolution inhibition and dissolution acceleration is remarkable, $\mathrm{B} / \mathrm{P}$ value can be designed higher, the photosensitivity is also higher and the quantity of exposure energy required for imaging is about $200 \mathrm{~mJ} / \mathrm{cm}^{2}$. In addition, it has good contrast and imaging film is easy to be taken off in concentrated alkaline liquor.

The PACs are widely used in photoresists for integrated circuit(IC), especially in photoresists for liquid crystal display(LCD) and photosensitive compositions for positive PS plates including heat-sensitive computer-to-plate(CTP) plates.

Keywords: photoactive compound (PAC); imaging performance; photoresist; PS plate; liquid crystal displayer(LCD)

\section{Introduction}

There have been previously a lot of studies and reports on DNQ series photoactive compounds(PAC) used for positive photoresists ${ }^{(1-3)}$. Based on industrial application and selection 2,1,5-diazonaphthoquinone sulfonic acid ester whose ballast is polyhydroxyl benzophenone is so far the best PAC for photoresists when exposed by $\mathrm{g}$ line and $\mathrm{i}$ line. As photoresists, the photosensitive compositions composed of this kind of PAC and novolac resins which are synthesized from $\mathrm{m}$-cresol and p-cresol have many advantages such as great thermal stability, high sensitivity when exposed by g line, good development latitude and resolving power, etc. So the PACs have played an important role in the manufacture of $64 \mathrm{k}$ and even $32 \mathrm{MB}$ silicone wafer and they are still used widely now. But the ballast of polyhydroxyl benzophenone which is an UV absorber has absorption in near UV region especially in deep UV region, so the bleaching performance of the
PACs synthesized from it is poor in near and deep UV region during exposure. The photodecomposition of the inner layer of the resist film will be influenced so the photosensitivity decreases even when exposed by i line (365nm). Usually deep UV line such as $254 \mathrm{~nm}$ or $248 \mathrm{~nm}$ is used to improve the resolving power of the resists, however the defect of the PACs is more evident as a consequence. In order to overcome the shortcomings, a series of non-polyhydroxyl PACs have been developed in recent years ${ }^{(2,4)}$, such as diazonaphthoquinone sulfonic esters of mono-alicyclic alcohols, poly-alicyclic alcohols and phenols compounds, but their comprehensive performances are not as good as the PACs synthesized from polyhydroxyl benzophenone. Especially in the dissolution inhibiting effect before exposure, dissolution accelerating effect after exposure and resolving power, polyhydroxyl series PACs have obvious predominance. 
The ballasts which are condensed from benzilic acid with phenolic compounds contain either inner-ester structure or carboxylic acid structure. The PACs synthesized from these ballasts containing inner-ester structure have several advantages, such as high photosensitivity, strong dissolution inhibiting effect before exposure, good dissolution accelerating effect after exposure, better bleaching performance in near and deep UV region, and easily film-taken off in concentrated alkaline liquor at room temperature. They have good prospects as normal positive photoresists, especially the positive photoresists for liquid crystal displayer and photo- and heat-sensitive compositions for printing plates.

\section{Experimental}

\subsection{Ballasts for the new PACs}

Five ballasts from formula 1 to 5 were all<smiles>O=C1Oc2cc(O)ccc2C1(c1ccccc1)c1ccccc1</smiles>

1<smiles>O=C1Oc2ccc(O)cc2C1(c1ccccc1)c1ccccc1</smiles>

3 condensed by benzilic acid with different polyhydroxyl benzene using p-toluene sulfonic acid or $\mathrm{H}_{2} \mathrm{SO}_{4}$ as catalyst and toluene or xylene as refluxing-dewater solvent. Toluene or xylene was distilled off after the volume of water separated during the course was equal to theoretical volume, then some distilled water was added and $\mathrm{PH}$ value of the system was adjusted to about 6 with sodium carbonate solution. The residual toluene or xylene was removed thoroughly by further heating azeotropism. The product and water were transferred to a beaker and filtered, the product was washed repeatedly, then water in the product was extracted completely, finally the product was dried at $60-80^{\circ} \mathrm{C}$. The synthesis and purification methods of the ballasts have been described in $\mathrm{CN}$ Patent $^{(5)}$.

Related performances and some data of the obtained ballasts are shown in table 1 .

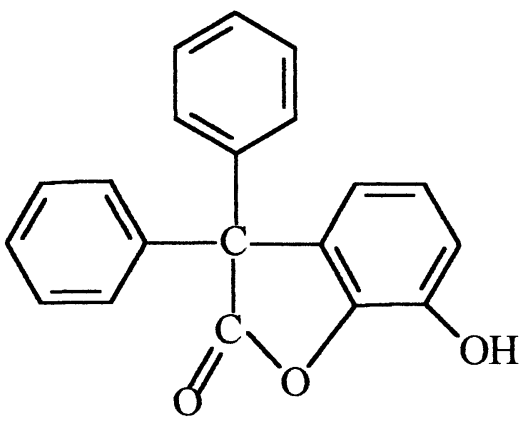

2<smiles>O=C1Oc2c(ccc(O)c2O)C1(c1ccccc1)c1ccccc1</smiles>

4 


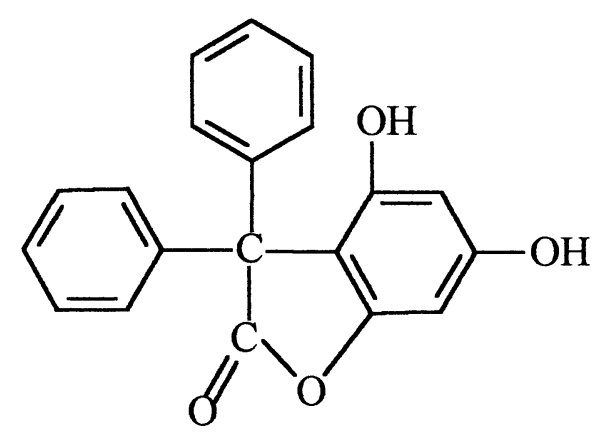

5

Tab.1 Performances of the synthesized ballasts

\begin{tabular}{|c|c|c|c|c|c|c|c|}
\hline Ballast & B-1 & B-2 & B-3 & B-4 & B-5 & B-6** & B-7*** \\
\hline M & 302 & 302 & 302 & 318 & 318 & 230 & 120 \\
\hline $\mathrm{M} / \mathrm{A}$ & 17.76 & 17.76 & 17.76 & 9.35 & 9.35 & 4.51 & 7.06 \\
\hline m.p. $\left({ }^{\circ} \mathrm{C}\right)$ & 172 & 125 & 184 & 186 & 210 & 144 & 120 \\
\hline $\begin{array}{l}\text { Abs(O.D.) } \\
\text { at 365nm }\end{array}$ & 0 & 0 & 0 & 0 & 0 & 0.9 & 0 \\
\hline $\begin{array}{l}\text { Abs(O.D.) } \\
\text { at } 248 \mathrm{~nm}\end{array}$ & 1.20 & 1.10 & 1.20 & 1.15 & 1.25 & $>4.00$ & $<1.10$ \\
\hline $1 \% \mathrm{NaOH}$ & Ds & Ds & Ds & $\mathbf{s}$ & $S$ & es & Es \\
\hline n-Heptane & I & I & I & i & I & i & I \\
\hline Xylene & Ds & Ds & Ds & ds & Ds & i & I \\
\hline Ketone & Es & Es & Es & es & Es & es & Es \\
\hline 1,4-Dioxane & Es & Es & Es & es & Es & es & Es \\
\hline $\begin{array}{l}\text { Ethyleneglycol } \\
\text { monoethyl ether }\end{array}$ & S & S & $S$ & es & Es & es & Es \\
\hline
\end{tabular}

* ds-difficultly soluble i-insoluble s-soluble es-easily soluble

** Synthesized from trihydroxy benzophenone

*** Synthesized from novolac and diazonaphtoquinone syulfonic acid chloride

\subsection{Synthesis of the PACs}

A series of PACs were respectively synthesized from each ballast and corresponding quantity diazonaphthoquinone sulfonic acid chloride with the traditional synthesis method of PAC. The solvent was acetone or 1,4-dioxane, volume of which was 4-6 times as much as the reactants volume. As catalyst, triethylamine or $10 \%$ sodium carbonate solution was added completely in one hour and the reaction temperature was controlled between $38-40^{\circ} \mathrm{C}$, then the reaction continued for 30 minutes. The reaction solution was poured in distilled water 10 times as much as volume of the solution and the $\mathrm{PH}$ value was adjusted to 5-6. After full precipitation the product was filtered and washed with distilled water repeatedly. At last the product was washed with iso-propanol and dried at low temperature or at vacuum. The related performances of the synthesized PACs are shown in table 2.

Tab. 2 Performances of the synthesized PACs

\begin{tabular}{|c|c|c|c|c|c|c|c|c|c|c|}
\hline PAC No. & 1 & 2 & 3 & $4(1)$ & $4(2)$ & $5(1)$ & $5(2)$ & $6(1)$ & $6(2)$ & 7 \\
\hline Appearance & \multicolumn{10}{|c|}{ yellow solid } \\
\hline Yield & $78 \%$ & $72 \%$ & $74 \%$ & $75 \%$ & $71 \%$ & $60 \%$ & $64 \%$ & $>90 \%$ & $>90 \%$ & $95 \%$ \\
\hline M.p. $\left({ }^{\circ} \mathrm{C}\right)$ & 130 & 130 & 130 & $>110$ & $>120$ & $>130$ & $>130$ & 130 & 130 & $>150$ \\
\hline $1 \% \mathrm{NaOH}$ & ds & ds & ds & ds & ds & ds & Ds & $\mathbf{s}$ & ds & $\mathrm{i}$ \\
\hline n-Heptane & $\mathrm{i}$ & $\mathrm{i}$ & $\mathrm{i}$ & $\mathrm{i}$ & $\mathrm{i}$ & $\mathrm{i}$ & I & $\mathrm{i}$ & $\mathrm{i}$ & $\mathrm{i}$ \\
\hline Isopropanol & ds & ds & ds & ds & ds & ds & Ds & $\mathbf{s}$ & ds & $\mathrm{i}$ \\
\hline Xylene & ds & ds & ds & ds & ds & ds & Ds & $\mathrm{i}$ & $\mathrm{i}$ & $\mathrm{i}$ \\
\hline Ketone & $\mathbf{s}$ & $\mathrm{s}$ & $\mathbf{s}$ & $\mathbf{s}$ & es & $\mathbf{s}$ & $\mathrm{S}$ & $\mathrm{s}$ & $\mathbf{s}$ & $\mathbf{s}$ \\
\hline 1,4-Dioxane & $\mathbf{s}$ & $\mathrm{s}$ & $\mathbf{s}$ & $\mathbf{s}$ & es & $\mathbf{s}$ & Es & $\mathbf{s}$ & $\mathbf{s}$ & $\mathbf{s}$ \\
\hline $\begin{array}{l}\text { Ethyleneglycol } \\
\text { monoethyl ether }\end{array}$ & $\mathbf{s}$ & $\mathbf{s}$ & $\mathbf{s}$ & $\mathbf{s}$ & es & $\mathbf{s}$ & S & $\mathbf{s}$ & $\mathbf{s}$ & $\mathbf{s}$ \\
\hline $\begin{array}{l}\text { Ethyleneglycol } \\
\text { monoethyl ether acetate }\end{array}$ & ds & ds & ds & ds & $\mathbf{S}$ & ds & $\mathrm{S}$ & es & es & es \\
\hline
\end{tabular}

*ds-difficultly soluble i-insoluble s-soluble es-easily soluble 


\subsection{Binder resin used for photosensitive} compositions

The binder resin being used is BTB-25 novolac which is developed and produced by Beijing Normal University combined with Tiancheng chemical technology company in Weihai City in Shandong Province. Some data of BTB-25 is shown as follows: $\mathrm{M}_{\mathrm{W}}=4000$; $\mathrm{M}_{\mathrm{W}} / \mathrm{M}_{\mathrm{N}}=3.6$; softening point temperature $115^{\circ} \mathrm{C}$; fog-muddy point( $\mathrm{PH}$ value) 11.4; the content of phenolic monomers $<1 \%$; condensation ratio in double ortho way 0.65 . The mixed phenolic resin which is condensed from m-cresol and p-cresol with formaldehyde can also be used as the binder resin.

\subsection{Analysis}

IR spectrum was measured with SHIMANZ IR 408 spectrometer by the way of film coating or tableting. UV spectrum was measured by HITACHI UV 240 spectrometer. NMR spectrometer was used to define the introduction position and microscopic structure of the product by DEPT135 treatment. It can determinate components of the product and content of major product when matched with GC-MS (gas chromatography-mass spectrometer).

The dissolution inhibiting/dissolution accelerating effect of the PAC is characterized with the optimum $M_{B} / M_{P}$ value (written in $B / P$ briefly) where $M_{B}$ is the binder resin mass and $M_{P}$ is the PAC mass. The higher $\mathrm{B} / \mathrm{P}$ value is, the stronger dissolution inhibiting effect is. The optimum $B / P$ value refers to the $B / P$ value at which the best image is obtained in practical developer. The maximum $B / P$ value is the $B / P$ value at which better image is gotten in permitted weak developer. The $\mathrm{M}_{\mathrm{C}} / \mathrm{M}_{\mathrm{DNQ}}$ value or $\left(\mathrm{M}_{\mathrm{C}} / \mathrm{M}_{\mathrm{DNQ}}\right)_{\max }$ value can also indicate the measurement of the dissolution inhibiting effect where $M_{C}$ is the whole mass of the photosensitive composition and $\mathrm{M}_{\mathrm{DNQ}}$ is the mass of DNQ groups in the photosensitive composition.

The relative photosensitivity of the PACs is represented by the most suitable exposure energy of each PAC, which is the exposure energy that is required for three steps to be washed out completely in practical developer after the photosensitive layer has been exposed with Kodak Photographic Step Tablet No.2. The less the exposure energy is, the higher the sensitivity is.

Other assessing methods are described in references 6-7.

\section{Results and Discussion}

3.1 Characteristics of the ballasts

Each molecule of this kind of ballast has three benzene rings, two of which have no polar substituted group and one contains both alkaline soluble phenolic hydroxyl group and phenolic inner-ester structure, so some properties in table 1 are determined: (1) $\mathrm{M} / \mathrm{A}$ value of the molecule is high, for example M/A value of B-1 is four times as high as $\mathrm{M} / \mathrm{A}$ value of trihydroxyl benzophenone. The dissolution inhibiting effect of the ballast itself is very strong in alkaline solution. (2) One hand, the inner-ester link in the molecule strengthens the dissolution inhibiting effect of the ballast because of an association reaction between carbonyl group of ester and phenolic hydroxyl group of the novolac, on the other hand inner-ester link is easily hydrolyzed in concentrated alkaline solution at room temperature, which benefits the photoresist image to be taken off finally. (3)The absorption of each ballast molecule in near and deep UV region is far less than that of trihydroxyl benzophenone, especially in nearby $365 \mathrm{~nm}$ region there is no absorption almost, so the bleaching performance of PAC synthesized from the ballast is good. The performances above mentioned are very ideal for the ballast of the new type PAC.

\subsection{Solvent performance of the PACs}

The PACs are easily soluble in common solvent for PS plates (including thermal-sensitive CTP plates) and the photoresists, for example, they are soluble or easily soluble in ketone, ethyleneglycol monoethyl ether acetate and 1,4-dioxane etc. They are difficultly soluble or insoluble in printing ink solvent such as petroleum series solvent or benzene series solvent. They also have resistances to ethanol or iso-propanol which is added in the moistening plates. The experiments indicate that anti-etching performance of the PACs is very strong in acid solution, such as $1 \% \mathrm{HCl}, \mathrm{HF}$ and $\mathrm{H}_{2} \mathrm{SO}_{4}$. Another remarkable performance is that they are hydrolyzed and taken off easily in $\mathrm{NaOH}$ solution with concentration higher than $3 \%$, although they are very difficult to be hydrolyzed in $1 \% \mathrm{NaOH}$ solution. The performances are ideal for PAC which is used in the resists or the printing plates.

3.3 Dissolution inhibiting/dissolution accelerating performance of the PACs

The dissolution inhibiting effect of the PACs in alkaline developer should be as strong as 
possible before exposure or heat scanning. It is expected that the dissolution accelerating effect gets strong to some extent in alkaline developer after exposure or scanning and wider development latitude can be obtained.

Tab.3 Dissolution inhibiting performances and calculation results of the PACs

\begin{tabular}{lcccccccccc}
\hline PAC No. & 1 & 2 & 3 & $4(1)$ & $4(2)$ & $5(1)$ & $5(2)$ & $6(1)$ & $6(2)$ & 7 \\
\hline $\mathrm{Mp}$ & 534 & 534 & 534 & 550 & 782 & 550 & 782 & 462 & 694 & 2128 \\
$\mathrm{~A}$ & 0 & 0 & 0 & 17 & 0 & 17 & 0 & 34 & 17 & 102 \\
$(\mathrm{M} / \mathrm{A})_{\mathrm{PAC}}$ & $\propto$ & $\propto$ & $\propto$ & 32.35 & $\propto$ & 32.35 & $\propto$ & 13.59 & 40.82 & 20.86 \\
$\mathrm{M}_{\mathrm{B}} / \mathrm{M}_{\mathrm{P}}(\mathrm{B} / \mathrm{P})$ & 4 & 3.5 & 4 & 3 & 5.5 & 3 & 5 & 2.5 & 4 & 2 \\
$(\mathrm{~B} / \mathrm{P})_{\max }$ & 5 & 4.5 & 5 & 4 & 8 & 4 & 6 & 3.5 & 5 & 3 \\
$\mathrm{M}_{\mathrm{DNQ}} / \mathrm{M}_{\mathrm{P}}$ & 0.44 & 0.44 & 0.44 & 0.42 & 0.60 & 0.42 & 0.60 & 0.50 & 0.67 & 0.44 \\
$\mathrm{M}_{\mathrm{C}} / \mathrm{M}_{\mathrm{DNQ}}$ & 11.46 & 10.31 & 11.46 & 9.44 & 10.91 & 9.44 & 10.07 & 6.94 & 7.45 & 6.85 \\
$\mathrm{RFR}$ & $>98 \%$ & $>98 \%$ & $>98 \%$ & $>90 \%$ & $>98 \%$ & $>90 \%$ & $>90 \%$ & $>90 \%$ & $>90 \%$ & $>90 \%$ \\
\hline
\end{tabular}

*RFR: residual film ratio in developer for $3 \mathrm{~min}$

Table 3 shows the experimental data and calculation results of related dissolution inhibiting effect of the PACs compared with PAC- 6 synthesized from trihydroxyl benzophenone. As for M/A value, when one hydroxyl group of each ballast molecule and trihydroxyl benzophenone introduces DNQ group, M/A values of PAC-1, PAC-2 and PAC-3 are all infinite and M/A values of PAC- 4(1), PAC-5(1) are both 32.35, but M/A value of PAC-6(1) is only 13.59 and $\mathrm{M} / \mathrm{A}$ value of PAC-6(2) which is synthesized with two DNQ groups being introduced to trihydroxyl benzophenone is just 40.82 . It is illustrated that $\mathrm{M} / \mathrm{A}$ value of the synthesized PAC increases if $\mathrm{M} / \mathrm{A}$ value of the ballast itself is high and the dissolution inhibiting effect of the PACs inevitably becomes strong. Previously, the dissolution inhibiting effect was measured by the dissolution inhibiting index $\mathrm{M}_{\mathrm{DNQ}} / \mathrm{M}_{\mathrm{PAC}}$, which is the mass ratio of DNQ groups to the molecular weight. It was generally considered that the higher the dissolution inhibiting index, the stronger the dissolution inhibiting effect of PAC. It is not the case in fact, $\mathrm{M}_{\mathrm{DNQ}} / \mathrm{M}_{\mathrm{PAC}}$ value just means the mass ratio of DNQ group to one PAC molecule as shown in table 3. High $\mathrm{M}_{\mathrm{DN}} \mathrm{Q} / \mathrm{M}_{\mathrm{PAC}}$ value indicates that the consumption of diazonaphthoquinone sulfonic acid chloride is great when $1 \mathrm{~mol}$ PAC is synthesized. The dissolution inhibiting/dissolution accelerating performance of PAC is assessed by selecting the best $M_{B} / M_{P}$ value $(B / P)$ and the permitted highest $\mathrm{B} / \mathrm{P}$ value after preparing photosensitive composition with each PAC and novolac binder resin. The higher the two values are, the stronger the dissolution inhibiting effect of the PAC is. The experiment data in table 3 indicate the values of the new PACs are higher than that of PAC- 6 at the same introduced ratio. The optimum $\mathrm{M}_{\mathrm{C}} / \mathrm{M}_{\mathrm{DNQ}}$ value and permitted maximum $\mathrm{M}_{\mathrm{C}} / \mathrm{M}_{\mathrm{DNQ}}$ value of the PACs are both higher than related values of PAC-6 at the same introduced ratio. When compared with PAC-7 (synthesized from novolac and diazonaphthaquinone sulfonic acid chloride) in the photosensitive compositions for PS plates, four above mentioned values of the new PACs are all higher. It is illustrated convincingly that the PACs behavior the strongest dissolution inhibiting effect before exposure scanning. Related experiment data and calculation results of the new PACs, trihydroxyl benzophnone series PACs and cresol phenolic resin series PAC after exposure are shown in table 4. Among these PACs, $(\mathrm{M} / \mathrm{A})_{\mathrm{AFT}}$ value of PAC-6 is the lowest, (M/A) $)_{\mathrm{AFT}}$ value of PAC-7 synthesized from phenolic resin is higher than that of PAC- 6 and $(\mathrm{M} / \mathrm{A})_{\mathrm{AFT}}$ values of the new PACs are the highest relatively. Because molecular weights of the new PACs are not very high, they are easily developed even in weaker alkaline developer and excess exposure does not lead to the loss of dot and line. 
Tab. 4 Related results about dissolution/accelerating effect

of the PACs after exposure

\begin{tabular}{lcccccccccc}
\hline PAC No. & 1 & 2 & 3 & $4(1)$ & $4(2)$ & $5(1)$ & $5(2)$ & $6(1)$ & $6(2)$ & 7 \\
\hline $\mathrm{M}_{\mathrm{AFT}}$ & 524 & 524 & 524 & 540 & 762 & 540 & 762 & 452 & 674 & 2088 \\
$\mathrm{~A}_{\mathrm{AFT}}$ & 45 & 45 & 45 & 62 & 90 & 62 & 90 & 79 & 107 & 282 \\
$(\mathrm{M} / \mathrm{A})_{\mathrm{AFT}}$ & 11.64 & 11.64 & 11.64 & 8.71 & 8.47 & 8.71 & 8.47 & 5.72 & 6.30 & 7.40 \\
$\mathrm{R}$ & $\propto$ & $\propto$ & $\propto$ & 3.71 & $\propto$ & 3.71 & $\propto$ & 2.38 & 6.48 & 2.82 \\
Developing & $15-30$ & $15-30$ & $15-30$ & $15-30$ & $15-30$ & $15-30$ & $15-30$ & $15-30$ & $15-30$ & $15-30$ \\
time(s) & no & No & no & no & No & no & no & No & no & no \\
Toning & & &
\end{tabular}

*(1)R:(M/A) PAd $_{\text {Pad }}(\mathrm{M} / \mathrm{A})_{\mathrm{AFT}}$ (2) thickness of film:1 $\mu \mathrm{m}$

According to related developing time in table 4 , the data of the photosensitive compositions containing the new PAC are within the controlled scope and the performances of the photosensitive compositions are at the same level with the resist compositions containing PAC-6. When (M/A) AFT value is high, it is worried that there is toning after development. According to the data in table 4 the misgivings can be dispelled. As for the time without gloss loss of the photosensitive composition film in the developer (residual film ratio is approximate to $100 \%$ ), the performances of the photosensitive compositions containing the new PAC are the best.

\subsection{Imaging performance of the PACs}

The imaging performance of the photosensitive composition composed of each new PAC and BTB-25 is shown in table 5. The most suitable imaging energy of PAC-1, PAC-3 and PAC-4 is close to that of PAC-6. The lowest decomposition energy of PAC-6(1) and PAC-4(1) is lower. The resolving power of PAC-6(2), PAC-1 and PAC-4(2) is higher than that of the other PACs. The image with $1 \mu \mathrm{m}$ line width can be obtained on the silicone wafer. As for anti-acid etching performance of the image, the majority of the photosensitive compositions containing the new PACs have stronger resistance to $\mathrm{HF}, \mathrm{H}_{2} \mathrm{SO}_{4}$, $\mathrm{HNO}_{3}$ and $\mathrm{H}_{3} \mathrm{PO}_{4}$. The PACs have excellent lipophilic as the photosensitive compositions for PS plates and have predominance as resists at two aspects of the bleaching performance in near and deep UV region and film-taken off performance in concentrated alkaline solution at room temperature compared with trihydroxyl benzophenone series PACs.

Tab. 5 Related results about imaging performance

\begin{tabular}{|c|c|c|c|c|c|c|c|c|c|c|c|}
\hline \multicolumn{2}{|l|}{ PAC.No. } & 1 & 2 & 3 & $4(1)$ & $4(2)$ & $5(1)$ & $5(2)$ & $6(1)$ & $6(2)$ & 7 \\
\hline \multicolumn{2}{|c|}{ Optim. exposed energy $\left(\mathrm{mJ} / \mathrm{cm}^{2}\right)$} & 250 & 250 & 250 & 250 & 240 & 250 & 240 & 240 & 240 & 300 \\
\hline \multicolumn{2}{|c|}{ Min. decom. energy $\left(<\mathrm{mJ} / \mathrm{cm}^{2}\right)$} & 120 & 120 & 120 & 100 & 120 & 100 & 120 & 100 & 120 & 150 \\
\hline \multirow{2}{*}{$\begin{array}{l}\text { Resolving } \\
\text { power }\end{array}$} & $\begin{array}{l}\text { Silicone } \\
\text { wafer }(\mu \mathrm{m})\end{array}$ & 1 & 1 & 1 & 1.5 & 1 & 1.5 & 1 & 1.2 & 1 & 2 \\
\hline & PS plate $(>1$ lpi) & 200 & 200 & 200 & 175 & 200 & 175 & 200 & 175 & 200 & 175 \\
\hline \multirow{2}{*}{$\begin{array}{l}\text { Anti-acid } \\
\text { etching }\end{array}$} & $\mathrm{HCl} \mathrm{HF}$ & $\mathrm{E}$ & $\mathrm{E}$ & $\mathrm{E}$ & G & $\mathrm{E}$ & G & $\mathrm{E}$ & $\mathrm{G}$ & $\mathrm{E}$ & $\mathrm{E}$ \\
\hline & $\begin{array}{ll}\mathrm{H}_{2} \mathrm{SO}_{4} & \mathrm{HNO}_{3} \\
\mathrm{H}_{3} \mathrm{PO}_{4} & \end{array}$ & $\mathrm{E}$ & G & $\mathrm{E}$ & G & $\mathrm{E}$ & G & $\mathrm{E}$ & G & $\mathrm{E}$ & G \\
\hline \multirow{2}{*}{\multicolumn{2}{|c|}{$\begin{array}{l}\text { Anti-plasma } \\
\text { Linophilic }\end{array}$}} & $\mathrm{E}$ & G & E & G & $\mathrm{E}$ & $\mathrm{E}$ & $\mathrm{E}$ & G & $\mathrm{E}$ & $\mathrm{E}$ \\
\hline \multirow{2}{*}{\multicolumn{2}{|c|}{$\begin{array}{l}\text { Lipophilic } \\
\text { Film-taken of }\end{array}$}} & $\mathrm{E}$ & $\mathrm{E}$ & $\mathrm{E}$ & G & $\mathrm{E}$ & G & $\mathrm{E}$ & G & G & $\mathrm{G}$ \\
\hline & & $\mathrm{E}$ & $\mathrm{E}$ & $E$ & $\mathrm{E}$ & $\mathrm{E}$ & $\mathrm{E}$ & E & $G$ & G & B \\
\hline
\end{tabular}

*E-excellent G-good B-bad

\section{Conclusion}

(1) The new PACs have higher M/A values and better dissolution inhibiting effect before exposure. When used as the photosensitive composition for PS plates or the resists, PAC with higher $B / P$ value and $M_{C} / M_{D N Q}$ value can be choosed.

(2) The photosensitivity, resolving power, dot reductivity, development latitude and image contrast of the photosensitive compositions containing the new PAC are identical with that of the photosensitive compositions containing trihydroxyl benzophenone series PAC. 
(3) When the new PACs are used as resists, the image film can be easily taken off in concentrated alkaline solution at room temperature after being etched.

(4) The bleaching performance of the photosensitive compositions containing the new PAC is better than that of trihydroxyl benzophenone series photosensitive compositions during exposure in near and deep UV region. The new PACs have great potentials as the positive resists, especially the resists for liquid crystal displayers and the photosensitive compositions for printing plates.

\section{References}

1.T. Yamaoka and H. Morita, "Photosensitive Resins", Kyoritsu Publ. in Japanese p43-44(1988) 2.Yu Shangxian, Tong Xiao, Gu Jiangnan, etc., Molecular design of PAC used for the positive photoresists:optimization of M/A value before and after photolysis, J. Photopolym. Sci. Thecnol. Vol.11, No.1, P89 96(1998)

3.L.F.Thompson, Introduction to Microlithography, Vol.2, No.19, P112-121

4.Yu Shangxian, Gu Jiangnan, Wang Fang, etc., Tung oil-pyrogallic acid resin(TPA) and its application to a positive photoresist, J. Photopolym. Sci. Thecnol. Vol.2, No.1, P51 56(1989)

5.Beijing Normal University, Yu Shangxian, Zou Yingquan, Shi Zhixiong, CN Patent, Application No.011236868(2001)

6. T. Yonezawa, "Introduction of PS Plates, Insatsu Gakkai Publ. in Japanese P109 111 (1993)

7. Yu Shangxian, Tong Xiao, Gu Jiangnan, etc., Design of the photosensitive compositions for positive photoresists, J. Photopolym. Sci. Technol. Vol.12, No.2, P319 324(1999) 